\title{
A Case of Lemierre's Syndrome in Association with Liver Abscess without Any Other Metastatic Lesions
}

\author{
Tomohiro Iwasaki ${ }^{1}$, Takashi Yamamoto ${ }^{2}$, Ken-ichiro Inoue ${ }^{1}$ and Ken-ichi Takaku ${ }^{1}$
}

\begin{abstract}
Lemierre's syndrome (LS) is characterized by pharyngitis followed by septicemia, internal jugular vein thrombophlebitis, and metastatic embolization in general. LS is commonly caused by Fusobacterium necrophorum. Herein, we present a case of LS with liver abscesses that presented as a sole metastatic lesion. We were not able to diagnose LS until Fusobacterium necrophorum was isolated due to the lack of the common involvement. Doripenem was effective against the pathologic features including the liver abscesses. LS should be taken into consideration when clinicians find liver abscesses following pharyngitis even when the common complications of LS are not detected.
\end{abstract}

Key words: Lemierre's syndrome, liver abscess, Fusobacterium necrophorum, carbapenem, pharyngitis

(Intern Med 51: 1419-1423, 2012)

(DOI: 10.2169/internalmedicine.51.7364)

\section{Introduction}

Lemierre's syndrome (LS) was first described by Courmont and Cade in 1900 (1) and it was well characterized by Lemierre in 1936 (2). LS is characterized by pharyngitis followed by septicemia, internal jugular vein (IJV) thrombophlebitis, and metastatic embolization to various organs, especially to the lungs $(3,4)$. The most common causative pathogen is Fusobacterium necrophorum. LS generally occurs in previously healthy young adults. In the pre-antibiotic era, LS was common and often fatal (2). However, there has been a substantial decrease in both the morbidity and mortality of LS along with the development and the widespread use of antibiotics for the treatment of pharyngeal infections. Nonetheless, recent reports have described the increasing incidence of LS (5-7). The cause of the increase is uncertain, but the increasingly judicious use of antimicrobial agents to treat pharyngeal infections and a change in the antibiotic susceptibility pattern of Fusobacterium necrophorum might be related to this increase (5). Although the mortality rate for LS has improved since the pre-antibiotic era, it is currently reported to be approximately $10 \%(3,4,8)$. Accordingly, early diagnosis and appropriate treatment are essential to improve the mortality rate. However, the diagnosis of LS is often not made until the isolation of Fusobacterium necrophorum from blood culture (4). Furthermore, when common complications such as IJV thrombophlebitis or pulmonary involvement are not overt, it becomes more difficult to diagnose LS in an expedient fashion.

Here, we describe a case of LS with liver abscesses that presented as a sole metastatic lesion, which is quite rare in LS (3). We were not able to diagnose LS based on clinical findings soon after the patient was admitted due to the lack of IJV thrombophlebitis and pulmonary involvement. Complementary examinations detected liver abscesses and sepsis induced by Fusobacterium necrophorum, which led to the diagnosis of LS. Doripenem (DRPM) was effective in treating this case, including the liver lesions.

\section{Case Report}

A previously healthy 44-year-old man visited a local clinic complaining of high fever and a sore throat. He was initially diagnosed with pharyngitis and was treated with a one-week course of oral cefdinir (CFDN). Thereafter, the sore throat improved, but a low-grade fever continued. Ten days after the onset of a sore throat, the patient developed a

${ }^{1}$ Department of Internal Medicine, Nanbugo General Hospital, Japan and ${ }^{2}$ Department of Respiratory Medicine, Nanbugo General Hospital, Japan

Received for publication January 20, 2012; Accepted for publication February 15, 2012

Correspondence to Dr. Tomohiro Iwasaki, iwattch_1125@yahoo.co.jp 
Table 1. Laboratory Data on Admission

\begin{tabular}{|c|c|c|c|c|c|}
\hline WBC & 15,190 & $/ \mu \mathrm{L}$ & PT & 12.8 & sec \\
\hline $\mathrm{RBC}$ & $473 \times 10^{4}$ & $/ \mu \mathrm{L}$ & PT-INR & 1.23 & \\
\hline Hemoglobin & 16.0 & $\mathrm{~g} / \mathrm{dL}$ & APTT & 39.3 & $\sec$ \\
\hline Hematocrit & 46.5 & $\%$ & FDP & $<10$ & $\mu \mathrm{g} / \mathrm{mL}$ \\
\hline Platelet & 61,000 & $/ \mu \mathrm{L}$ & Immunoglobulin $\mathrm{G}$ & 1,548 & $\mathrm{mg} / \mathrm{dL}$ \\
\hline Total protein & 6.1 & $\mathrm{~g} / \mathrm{dL}$ & Immunoglobulin A & 215 & $\mathrm{mg} / \mathrm{dL}$ \\
\hline Albumin & 3.5 & $\mathrm{~g} / \mathrm{dL}$ & Immunoglobulin $\mathrm{M}$ & 196 & $\mathrm{mg} / \mathrm{dL}$ \\
\hline BUN & 23 & $\mathrm{mg} / \mathrm{dL}$ & & & \\
\hline Creatinine & 1.2 & $\mathrm{mg} / \mathrm{dL}$ & \multicolumn{3}{|c|}{ Entamoeba histolytica antibody } \\
\hline Total Bilirubin & 2.4 & $\mathrm{mg} / \mathrm{dL}$ & \multicolumn{3}{|c|}{ negative } \\
\hline AST & 76 & IU/L & \multicolumn{3}{|c|}{ Dipstick urine examination } \\
\hline ALT & 160 & IU/L & \multicolumn{3}{|c|}{$2+$ proteinuria } \\
\hline $\mathrm{LDH}$ & 265 & $\mathrm{mg} / \mathrm{dL}$ & \multicolumn{3}{|c|}{$2+$ hematuria } \\
\hline ALP & 679 & $\mathrm{IU} / \mathrm{L}$ & \multicolumn{3}{|l|}{ On urine microscopy } \\
\hline$\gamma$-GTP & 697 & $\mathrm{IU} / \mathrm{L}$ & \multicolumn{3}{|c|}{ RBC 1-4 / HPF } \\
\hline CRP & 25.0 & $\mathrm{mg} / \mathrm{dL}$ & \multicolumn{3}{|c|}{$\mathrm{WBC}<1 / \mathrm{HPF}$} \\
\hline
\end{tabular}

WBC: white blood cell count, RBC: red blood cell count, BUN: blood urea nitrogen, AST: aspartate aminotransferase, ALT: alanine aminotransferase, LDH: lactate dehydrogenase, ALP: alkaline phosphatase, $\gamma$-GTP: $\gamma$-glutamyltranspeptidase, CRP: C-reactive protein, PT: prothrombin time, INR: international normalized ratio, APTT: activated partial thromboplastin time, FDP: fibrin and fibrinogen degradation products, HPF: high-power field
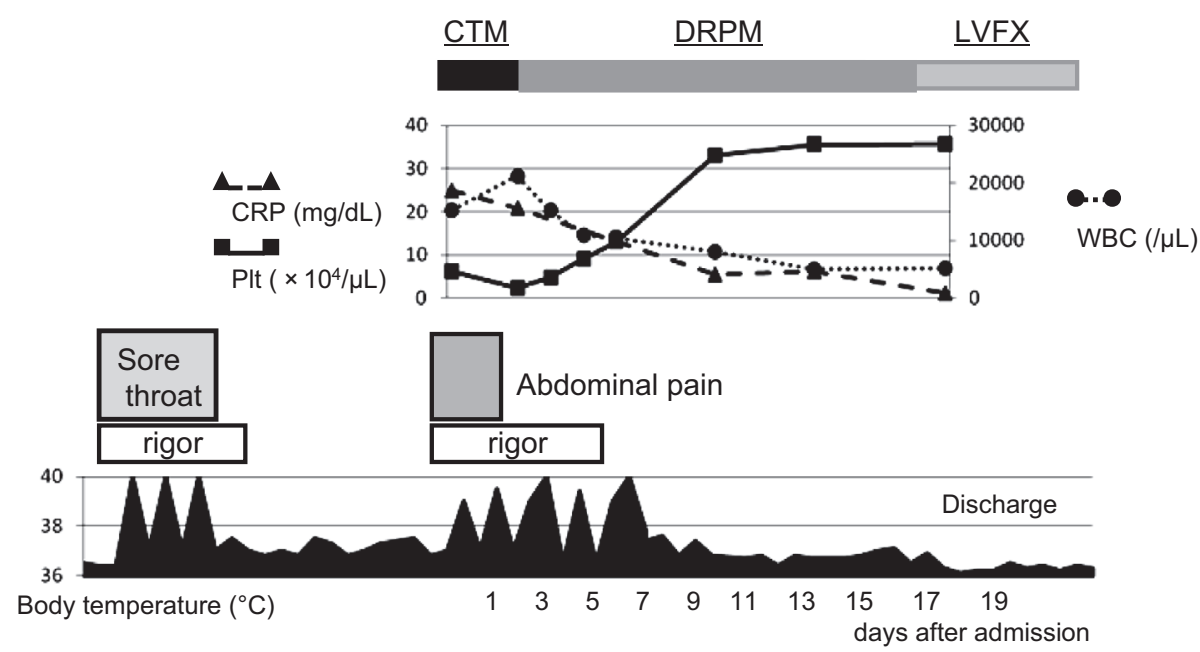

Figure 1. Schematic diagram of the patient's clinical course. High-grade fever appeared after admission but was alleviated by treatment with Doripenem (DRPM). A coagulation disturbance including thrombocytopenia was successfully treated.

high fever. Thirteen days after the onset of this sore throat, he visited another clinic and was referred to our hospital for further examination and treatment.

At the time of admission, the patient was $173 \mathrm{~cm}$ tall and weighed $82 \mathrm{~kg}$. He looked unwell and had a blood pressure of $98 / 59 \mathrm{mmHg}$, a pulse rate of $120 / \mathrm{min}$, a temperature of $38.9^{\circ} \mathrm{C}$ and a percutaneous oxygen saturation of $97 \%$. He was clearly conscious and was not icteric. The patient did not have a sore throat, neck tenderness or respiratory symptoms. The oropharynx appeared normal. Abdominal examination revealed mild right hypochondrial pain. Examinations of the respiratory and cardiovascular systems revealed no abnormality. Laboratory data on admission (Table 1) revealed a strong inflammatory reaction involving $25.0 \mathrm{mg} / \mathrm{dL}$ of C-reactive protein (CRP), thrombocytopenia, the elevation of liver enzymes and mild renal dysfunction. The clinical course is shown in Fig. 1. At first, the focus of the infection could not be identified. Then, a viral infection was suspected for the fever due to the mild liver injury and thrombocytopenia observed. However, certain bacterial infections, such as a urinary tract infection, were also simultaneously suspected because the white blood cell count (WBC) and the CRP level were increased. The patient was therefore intravenously treated with cefotiam (CTM) after a blood culture was obtained. After the patient was admitted, the degree of right hypochondrial pain improved, but the fever spike continued with rigor despite the administration of CTM. On the third hospital day, laboratory tests revealed sustained high-grade inflammation (WBC 21,180/ $\mu \mathrm{L}$, CRP $20.9 \mathrm{mg} /$ $\mathrm{dL}$ ), marked thrombocytopenia (platelets $23,000 / \mu \mathrm{L}$ ) and im- 


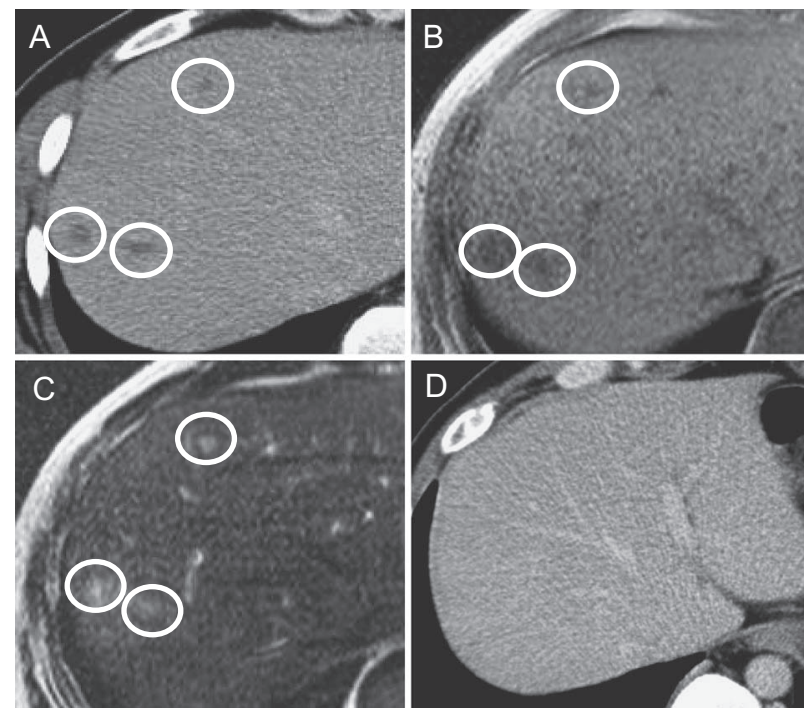

Figure 2. Imaging examinations. (A) Computed tomography (CT) revealed multiple small, low-density lesions in the liver. $(B, C)$ In the magnetic resonance images, these lesions exhibited hypointensity on T1-weighted images (B) and hyperintensity on T2-weighted images (C). (D) On the seventeenth hospital day, liver abscesses were no longer detectable by CT.

pairment of coagulation status (fibrin and fibrinogen degradation products: $10-19 \mu \mathrm{g} / \mathrm{mL}$, anti-thrombin III 59\%). Subsequent abdominal computed tomography (CT) revealed multiple small, low-density lesions in the liver (Fig. 2A), which were visualized as small, low-echoic lesions on abdominal ultrasonography. Magnetic resonance imaging (MRI) showed that these lesions exhibited hypointensity on T1-weighted images (Fig. 2B) and hyperintensity on T2weighted images (Fig. 2C). These findings were compatible with the existence of multiple liver abscesses. Entamoeba histolytica antibody staining was negative. The liver abscesses were considered to have caused the fever. In addition, disseminated intravascular coagulation (DIC) was indicated based on scoring criteria established by the Japanese Association for Acute Medicine for DIC. Taking these clinical findings into consideration, the antibiotic was changed to DRPM, and intravenous immunoglobulin therapy was initiated. Gabexate mesylate, a transfusion of platelets and human anti-thrombin III were also administered for the treatment of DIC. These treatments were effective and gradually ameliorated the patient's condition. On the tenth day of the patient's hospital stay, Fusobacterium necrophorum was isolated from the blood culture drawn on the second hospital day. At last, the patient was diagnosed LS according to the definition reported by Riordan (3), although Doppler ultrasonography did not detect IJV thrombophlebitis. The isolated Fusobacterium necrophorum was sensitive to ampicillin, pipercillin, cefazolin, sulbactem/cefoperazone, cefotaxime, ceftazidime, imipenem (IPM), meropenem (MEPM), DRPM, clindamycin (CLDM), clarithromycin, and levofloxacin (LVFX), and resistant toteicoplanin and vancomycin. Based on this microbial analysis, we continued treatment with DRPM. On the seventeenth hospital day, CT showed that the liver abscesses had disappeared (Fig. 2D). On the nineteenth hospital day, the patient was switched from treatment with antibiotics to treatment with oral LVFX. The strain of Fusobacterium necrophorum that had infected this patient was indeed sensitive to oral LVFX. The patient was discharged and no infectious symptoms have been observed since discharge.

\section{Discussion}

The definition of LS has not yet been established. Riordan reviewed numerous published cases of LS and established the following definition (3): (i) history of angina in the preceding 4 weeks or compatible clinical findings, (ii) evidence of metastatic lesions, (iii) evidence of IJV thrombophlebitis or isolation of Fusobacterium necrophorum or Fusobacterium spp. from blood cultures or a normal sterile site. Early diagnosis and prompt treatment with appropriate antibiotics are important to reduce the likelihood of mortality $(4,9)$. Chirinos et al. reported that in $69.7 \%$ of LS cases, the first clue to the diagnosis was the finding of Fusobacterium necrophorum in culture results rather than clinical signs or symptoms (4), which indicates that many clinicians will not be able to diagnose LS based on early clinical features. Fusobacterium necrophorum usually takes 2 to 7 days to grow in culture (10); therefore, fatal outcomes may result if proper treatment is not administered until the isolation of Fusobacterium necrophorum. In the present case, a diagnosis of LS could not be made during the early post-admission period. Reports based on the results of blood culture confirmed this diagnosis on the tenth hospital day. In particular, the lack of common complications, such as IJV thrombophlebitis or pulmonary involvement, resulted in a delayed diagnosis. LS should be taken into consideration when a previously healthy adult presents with acute sepsis following pharyngeal infection, despite the lack of any common complications associated with LS.

Septic embolizations of LS occur in various organs. Typically, IJV thrombophlebitis can give rise to septic emboli. However, IJV thrombophlebitis is only documented in 59$72 \%$ of patients with LS $(3,4)$. In such cases, septic emboli may spread via microvessels near the pharynx that are upstream of IJV, such as the tonsillar vein and the peritonsillar vein. On the other hand, liver abscess, which was the only metastatic complication in this case, is rare and identified in only $2-4 \%$ of LS cases $(3,4)$. A PubMed search revealed 13 reports of LS with liver abscess (Table 2). Liver abscesses are often multiple rather than solitary. Liver dysfunction was detected frequently (in 10 of these 13 cases). However, abnormal liver function is not a characteristic finding of liver abscess in the context of LS because it is found in 30-50\% of LS cases regardless of the existence of a liver abscess $(3,4)$. Among these 13 cases, there were only 5 in which the liver abscess was the only metastatic lesion, which implies that liver abscess as the sole systemic mani- 
Table 2. Clinical Features of Reported Cases of Lemierre's Syndrome with Liver Abscess

\begin{tabular}{lccccccc}
\hline $\begin{array}{c}\text { Number } \\
\text { (ref) }\end{array}$ & $\begin{array}{c}\text { Age } \\
\text { /sex }\end{array}$ & $\begin{array}{c}\text { Antibiotics } \\
\text { for pharyngitis }\end{array}$ & $\begin{array}{c}\text { Liver } \\
\text { dysfunction }\end{array}$ & $\begin{array}{c}\text { Morphology of } \\
\text { liver abscess }\end{array}$ & $\begin{array}{c}\text { IJV } \\
\text { thrombophlebitis }\end{array}$ & $\begin{array}{c}\text { Other metastatic } \\
\text { lesions }\end{array}$ & $\begin{array}{c}\text { Antibiotics } \\
\text { given }\end{array}$ \\
\hline 1. (16) & $11 \mathrm{y} / \mathrm{NR}$ & NR & Yes & NR & NR & NR & CLDM+MTZ+LMOX \\
2. (17) & $17 \mathrm{y} / \mathrm{NR}$ & NR & Yes & multiple & No & empyema & CXM+GM+MTZ \\
3. (11) & $17 \mathrm{y} / \mathrm{F}$ & No & Yes & multiple & Yes & lung & NFPC+CTRX+MTZ \\
4. (18) & $18 \mathrm{y} / \mathrm{M}$ & No & Yes & single & Yes & lung, empyema & CFPM+MTZ \\
5. (19) & $18 \mathrm{y} / \mathrm{M}$ & PC & Yes & multiple & No & lung & CXM+MTZ \\
6. (20) & $19 \mathrm{y} / \mathrm{F}$ & AMPC & Yes & multiple & No & empyema & PC+MTZ+CPFX \\
7. (21) & $32 \mathrm{y} / \mathrm{M}$ & No & NR & single & Yes & lung, empyema & CLDM+MEPM+TEIC \\
8. (15) & $70 \mathrm{y} / \mathrm{F}$ & CAM & Yes & single & Yes & lung, thyroid & IPM+MTZ \\
9. (22) & $8 \mathrm{~m} / \mathrm{M}$ & PC & No & multiple & No & No & ABPC+GM+MTZ \\
10. (23) & $5 \mathrm{y} / \mathrm{F}$ & PC & Yes & single & No & No & PC+CP \\
11. (24) & $21 \mathrm{y} / \mathrm{M}$ & AMPC & No & multiple & No & No & TAZ+MTZ \\
12. (25) & $22 \mathrm{y} / \mathrm{M}$ & No & Yes & multiple & No & No & GM+MTZ+CTRX+CLM \\
13. (this case) & $44 \mathrm{y} / \mathrm{M}$ & CFDN & Yes & multiple & No & No & DRPM \\
\hline
\end{tabular}

PC: penicillin, AMPC: amoxicillin, CAM: clarithromycin, CFDN: cefdinir, MTZ: metronidazole, LMOX: moxalactam, CXM: cefuroxime, GM: gentamicin, NFPC: nafcillin, CTRX: ceftriaxone, CFPM: cefepime, CPFX: ciprofloxacin, CLDM: clindamycin, MEPM: meropenem, TEIC: teicoplanin, IPM: imipenem, ABPC: ampicillin, CP: chloramphenicol, TAZ: tazobactam, DRPM: doripenem, NR: non-referred, Ref: reference, IJV: internal jugular vein

festation in LS patients is quite rare. It is not clear why septic emboli were found only in the liver. Chirinos et al. found that early antibiotic treatment could prevent the metastatic spread of infection through embolization (4). In 4 of these 5 cases, antibiotics were administered to treat pharyngitis; therefore, it is possible to assume that the prescription of antibiotics for pharyngitis prevented the development of septic emboli in all organs other than the liver. It is unclear why this approach to treatment was not able to prevent the development of a liver abscess. Nonetheless, administering antibiotic treatment to all patients with pharyngitis to prevent the spread of septic emboli in case of LS is not recommended because LS is still rare and the abuse of antibiotics induces resistance. Therefore, when clinicians examine a previously healthy patient with a liver abscess after pharyngitis, even if liver pathology represents the only extrapharyngitic lesion, it is necessary to consider the possibility of LS and treat the patient with the appropriate antibiotics in an expedient fashion. Regarding therapeutic interventions, drainage of the liver abscess was performed in 8 of these 13 cases. According to Stallworth and Carroll, drainage of any LS abscesses is generally necessary for successful treatment (11). In the present case, all liver abscesses were small, and there were no drainable lesions.

Fusobacterium necrophorum is usually susceptible to penicillin, CLDM, metronidazole (MTZ), and chloramphenicol. However, the resistance rates of Fusobacterium necrophorum to penicillin and macrolides were reported to be as high as $22 \%$ and $66 \%$, respectively $(7,12,13)$. The strain of Fusobacterium necrophorum isolated in the case described here was sensitive to the drugs administered. Furthermore, in LS, mixed infections involving organisms such as oral streptococci may occur (10). Therefore, combination therapy with penicillin and MTZ is generally recommended (3). Recently, there have been several reports that combination therapy with carbapenems and MTZ or CLDM is effective in the treatment of $\operatorname{LS}(14,15)$. However, the efficiency of monotherapy with carbapenems for LS has not been reported. In the present case, we initially treated with CTM, but CTM was not effective. Then, DRPM was substituted for CTM, which led to an improvement in the patient's overall clinical features. In this case, when Fusobacterium necrophorum, which was sensitive to DRPM, had been isolated on the tenth hospital day, the patient had already improved, and the levels of inflammatory markers were almost normal. Therefore, we did not supplement the patient's treatment regimen with MTZ or CLDM. Furthermore, carbapenems have broad-spectrum activity against grampositive, gram-negative, and anaerobic bacteria. Taken together, these findings suggest that clinicians might be able to treat patients suspected of carrying LS with carbapenems alone.

Septicemic illness caused by Fusobacterium necrophorum, called as necrobacillosis $(3,26)$, arises from not only pharynx, but also the oral cavity, the gastrointestinal tract and the female genital tract (6). There are a few cases of necrobacillosis with no clear sources $(3,26)$. Nozawa et al. reported a case of liver abscess infected with Fusobacterium necrophorum without pharyngeal infections or IJV thrombosis (27). Furthermore, according to Nohrstrom et al., necro- 
bacillosis may be accompanied by abscesses without any apparent venous thrombosis (28). Therefore, when a patient presents with any abscesses of unknown origin, we clinicians should also take necrobacillosis into consideration and treat the patient with the appropriate antibiotics despite the lack of pharyngitis or venous thrombosis.

\section{Conclusion}

We report a case of LS with liver abscess as the sole septic metastasis. DRPM was effective in treating the pathologic features of this condition including liver abscesses. LS is a rare disease but it may be fatal without prompt and appropriate treatment. LS should be taken into consideration when examining a patient with septic illness and liver abscess after pharyngitis despite the lack of common complications such as IJV thrombophlebitis or pulmonary lesions. In addition, monotherapy with carbapenems may be effective in treating LS.

The authors state that they have no Conflict of Interest (COI).

\section{References}

1. Golpe R, Marin B, Alonso M. Lemierre's syndrome (necrobacillosis). Posgrad Med J 75: 141-144, 1999.

2. Lemierre A. On certain septicemias due to anaerobic organisms. Lancet 1: 701-703, 1936.

3. Riordan T. Human infection with Fusobacterium necrophorum (Necrobacillosis), with a focus on Lemierre's syndrome. Clin Microbiol Rev 20: 622-659, 2007.

4. Chirinos JA, Lichtstein DM, Garcia J, Tamariz LJ. The evolution of Lemierre syndrome: report of 2 cases and review of the literature. Medicine 81: 458-465, 2002.

5. Ramirez S, Hild TG, Rudolph $\mathrm{CN}$, et al. Increased diagnosis of Lemierre syndrome and other Fusobacterium necrophorum infections at a children's hospital. Pediatrics 112: e380-e385, 2003.

6. Hagelskjaer LH, Prag J, Malczynski J, Kristensen JH. Incidence and clinical epidemiology of necrobacillosis, including Lemierre's syndrome, in Denmark 1990-1995. Eur J Clin Microbiol Infect Dis 17: e561-e565, 1998.

7. Brazier JS, Hall V, Yusuf E, Duerden BI. Fusobacterium necrophorum infections in England and Wales 1990-2000. J Med Microbiol 51: 269-272, 2002.

8. Gowan RT, Mehran RJ, Cardinal P, Jones G. Thoracic complications of Lemierre syndrome. Can Respir 7: 481-485, 2000.

9. Lu MD, Vasavada Z, Tanner C. Lemierre syndrome following oropharyngeal infection: a case series. J Am Board Fam Med 22: 7983, 2009.

10. Riordan T, Wilson M. Lemierre's syndrome: more than a histori- cal curiosa. Postgrad Med J 80: 328-334, 2004

11. Stallworth JR, Carroll JM. Lemierre's syndrome: new insights into an old disease. Clin Pediatr (Phila) 36: 715-717, 1997.

12. Appelbaum PC, Spangler SK, Jacobs MR. $\beta$-lactamase production and susceptibilities to amoxicillin, amoxicillin-clavulanate, ticarcillin, ticarcillin-clavulanate, cefoxitin, imipenem, and metronidazole of 320 non-Bacteroides fragilis Bacteroides isolates and 129 Fusobacteria from 28 U.S. centers. Antimicrob Agents Chemother 34: 1546-1550, 1990.

13. Baquero F, Reig M. Resistance of anaerobic bacteria to antimicrobial agents in Spain. Eur J Clin Microbiol Infect Dis 11: 10161020, 1992.

14. Yoshikawa H, Suzuki M, Nemoto N, et al. Internal jugular thrombophlebitis caused by dermal infection. Intern Med 50: 447-450, 2011.

15. Kara E, Sakarya A, Keles C, Borand H, Pekindil G, Goktan C. Case of Lemierre's syndrome presenting with thyroid abscess. Eur J Clin Microbiol Infect Dis 23: 570-572, 2004.

16. Bilfinger TV, Hayden CK, Oldham KT, Lobe TE. Pyogenic liver abscesses in nonimmunocompromised children. South Med J 79: 37-40, 1986.

17. Koay CB, Heyworth $T$, Burden P. Lemierre syndrome- - a forgotten complication of acute tonsillitis. J Laryngol Otol 109: 657661, 1995.

18. Narsinghani U, Schmidt MB, Jacobs RF, Anand KS. Radiological case of the month: Lemierre syndrome. Arch Pediatr Adolesc Med 155: 965-966, 2001.

19. Thoufeeq MH, Salloum W, Win SS, Sinha L. Lemierre's syndrome secondary to Fusobacterium infection, a rare cause of hepatic abscess. Acta Gastroenterol Belg 72: 444-446, 2009.

20. Clarke MG, Kennedy NJ, Kennedy K. Serious consequences of a sore throat. Ann R Coll Surg Engl 85: 242-244, 2003.

21. Morizono S, Enjoji M, Sonoda $\mathrm{N}$, et al. Lemierre's syndrome: Porphyromonas asaccharolytica as a putative pathogen. Intern Med 44: 350-353, 2005.

22. Embree JE, Williams T, Law BJ. Hepatic abscesses in a child caused by Fusobacterium necrophorum. Pediatr Infect Dis $\mathrm{J}$ 7: 359-360, 1988.

23. Harrington E, Bleicher MA. Cryptogenic hepatic abscess in two uncompromised children. J Pediatr Surg 15: 660-662, 1980.

24. David H. A 21-year-old man with fever and abdominal pain after recent peritonsillar abscess drainage. Am J Emerg Med 27: 515. e3-4, 2009.

25. Thatcher P. Hepatic abscesses caused by Fusobacterium necrophorum as part of the Lemierre syndrome. J Clin Gastroenterol 37: 196-197, 2003.

26. Eykyn SJ. Necrobacillosis. Scand J Infect Dis Suppl 62: 41-46, 1089.

27. Nozawa Y, Joshita S, Fukushima M, et al. A case of pyogenic liver abscess infected with Fusobacterium necrophorum depicted by microscopy and confirmed by tissue culture. Intern Med 50: 1815-1819, 2011.

28. Nohrstrom E, Mattila T, Pettila V, et al. Clinicial spectrum of bacteraemic Fusobacterium infections: from septic shock to nosocomial bacteraemia. Scand J Infect Dis 43: 463-470, 2011.

(C) 2012 The Japanese Society of Internal Medicine

http://www.naika.or.jp/imindex.html 